\title{
Die Wirkungsmacht der Ökonomie
}

\author{
Unter den heutigen wirtschaftlichen und gesellschaftlichen Rahmenbedingungen \\ scheint sich sowohl der Verkehr als auch die Entwicklung der Städte selbst \\ gegen die (europäische) Stadt zu richten. Dazu tragen in nicht unerheblichem \\ Maße ökonomische Prozesse bei, denn Verkehrs- und Wirtschaftssystem sind \\ eng miteinander verflochten, obgleich sie auf unterschiedlichen räumlichen \\ Ebenen verschiedenartige Funktionen übernehmen. Diesem Zusammenspiel wird \\ sowohl in der Theorie als auch in der Planungspraxis bisher zu wenig Bedeu- \\ tung beigemessen.
}

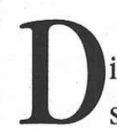
Von Heike Flämig ie Definition dessen, was unter Stadt verstanden werden kann, hat sich im Laufe der Zeit verändert. Früher war die Stadt Ort der Zuflucht, des Schutzes und der Rechte der Bürger, sichergestellt durch eine baulich-räumliche Trennung von der Umwelt, die Befestigungsmauern. Diese trugen zugleich auch dazu bei, dass Städte eine dichte und kompakte Baustruktur aufwiesen.

Auch heute noch wird mit der Stadt die räumliche Konzentration von Menschen und Ressourcen verbunden. Die Stadt als Kristallisationskern umfasst vor allem wirtschaftliche Aktivitäten und bündelt zugleich eine Vielzahl gesellschaftlicher Funktionen, wie Wohnen und Aufenthalt, Arbeiten und Ausbildung, Kultur und Freizeitgestaltung, Verwaltung und Gesundheitswesen sowie Versorgung mit Gütern und Dienstleistungen. Eines der zentralen Wesensmerkmale einer Stadt ist gerade diese Multifunktionalität. Hier laufen mannigfaltige ökonomische, soziale, politische und kulturelle Prozesse zusammen und beeinflussen sich gegenseitig.

Eine eindeutige Abgrenzung der Stadt als Einheit und damit die Lokalisierung des Wirkungsgebietes dieser Prozesse und Funktionen ist nur noch schwer möglich: Es gibt kaum noch geographisch-naturräumliche oder baulich-räumliche Grenzen; lediglich an einigen Stellen wirken (noch) administrative Grenzen (1). Dies gilt ebenso für die Quantität und Qualität von Arbeitsplätzen wie für die Breite und Tiefe der Versorgungsmöglichkeiten und nicht zuletzt für die Steuerungskraft von öffentlichen Leistungen. Damit wird die räumliche Verortung von Funktionen bzw. Prozessen zunehmend erschwert, die Grenzen zwischen Stadt und Umland zerfließen.
Hierfür sind gleichermaßen zwei Subsysteme verantwortlich: Erstens das Verkehrssystem, das durch den exzessiven Ausbau der Infrastruktur, die zunehmende Motorisierung und die abnehmenden Transportkosten zu einer veränderten Bedeutung von Mobilität, Entfernung und Erreichbarkeit beigetragen hat. Zweitens das Wirtschaftssystem, dessen Entwicklung zu einer globalen Ökonomie mit flexiblen Produktionskonzepten und neuen Logistikstrategien das innere und äußere Beziehungsgefiige der Städte stark verändert hat.

\section{Stadt und Verkehr}

Die wirtschaftlichen, administrativen und kulturellen Funktionen einer Stadt setzen den Austausch von Personen, Materialien (Güter, Waren) und Informationen und deren Mobilität voraus. Das Verkehrssystem schafft dafür den Rahmen und beeinflusst in hohem Maße die Multifunktionalität und Entwicklungsfähigkeit von Städten (2). Umgekehrt wird in Agglomerationen das Verkehrssystem stark durch Merkmale der städtischen Nutzungen, wie bauliche Dichte, Nutzungsmischung und (Poly-)Zentralität geprägt (3).

So können für die jüngere Vergangenheit jedem Stadttypus dominierende Verkehrsmittel zugeordnet werden: In der mittelalterlichen Stadt erfolgte die Fortbewegung vornehmlich zu Fuß oder Pferd. Dementsprechend war die Stadt dicht, kompakt und kleinteilig gemischt. Durch das zunehmende Bevölkerungswachstum, die politisch-gesellschaftlichen Veränderungen und die Verarmung der Landbevölkerung entstand ein neuer Siedlungsdruck, der vor allem durch (waffen)technische Entwicklungen eine neue Stadtstruktur entstehen ließ. Für die Stadterweiterung wurden die alten Befestigungsanlagen nieder geschliffen; sie wurden zu Straßen oder Bahnanlagen umfunktioniert. Der zunehmende Ausbau des Verkehrssystems stärkte die Städte als Verkehrsknotenpunkte und Handelszentren (4). In den Gründerjahren führte die zunehmende Industrialisierung und die netzartige Erschließung Deutschlands mit der Eisenbahn zu neuen Siedlungsstrukturen. Die Fabriken siedelten sich entlang der schienengebundenen Massenverkehrsmittel an.

Mit zunehmender Verbreitung des Automobils konnten der Raum flexibel erschlossen und Reisezeiten verkürzt werden. Eine flächenhafte Siedlungsentwicklung setzte ein, die die Innovationen in der Kommunikationstechnologie noch begünstigte.

Die zunehmende Motorisierung, die relative Abnahme der Transportkosten im Vergleich zum Einkommen und zum Wert des transportierten Gutes und der weitere Ausbau der Verkehrsinfrastruktur lassen den Verkehr weiter wachsen. Dies fuihrt vor allem in den Städten zu hohen Nutzungskonflikten und spezifischen Problemlagen, wie Flächenknappheit, erhöhte Luftverschmutzung und Lärmbelastung.

Ökologische wie ökonomische Faktoren tragen gleichzeitig auch zu veränderten siedlungsstrukturellen Entwicklungen bei. Die Bevölkerung sowie die Produktions- und Versorgungsfunktion zieht es aus den Innenstädten an die Verdichtungsränder, wie es die wachsenden regionalen Pendlerbeziehungen und Kaufkraftströme eindrucksvoll belegen. Aus Städten werden Stadtregionen.

\section{Sozio-ökonomische Faktoren der Stadtentwicklung}

Zur Unterscheidung von und zwischen Regionen, Städten oder Stadtquartieren werden zumeist unterschiedliche Ausprägungen der durch die Bewohner geprägten Merkmale Größe, Dichte und Heterogenität sowie deren Zusammenwirken herangezogen (5). Wie sich diese Merkmale verändern, welche Faktoren dabei eine entscheidende Rolle spielen und welche unterschiedlichen räumlichen Entwicklungen damit einhergehen, ist Gegenstand der Theorien zur Raumwirtschaft und Stadtentwicklung. Friedrichs hat aus diesen Theorien drei Faktoren herausdestilliert, die vorrangiges Untersuchungsfeld und damit Hintergrund für Erklärungsansätze von Stadtentwicklung sein können (6): Wirtschaftliche Veränderungen, gesellschaftliche Trends und die Nutzung neuer Technologien. Diese schlagen sich 
in der Stadt- und Raumstruktur nieder, wobei zwischen diesen drei Faktoren Wechselwirkungsverhältnisse bestehen.

Wird der Wirtschaft bei der (Stadt-) Entwicklung eine zentrale Rolle anerkannt, spielen Faktoren wie Verkehrsinfrastrukturausstattung, Agglomerationsvorteile und Transportkosten für die Stadt- und Raumentwicklung traditionell eine wichtige Rolle. Ein Blick auf die Entwicklungen in der jüngsten Vergangenheit zeigt jedoch, dass sich die Rahmenbedingungen stark verändert haben. Die Transportkosten haben rapide abgenommen und die Verfügbarkeit von Infrastruktur ist nahezu ubiquitär. Durch den Einsatz der neuen Informations- und Kommunikationstechnologien, durch die zunehmende Motorisierung und durch neue Formen von inner- und zwischenbetrieblicher Arbeitsteilung scheint der Erklärungsgehalt der klassischen Theorieansätze, der primär auf Transportkosten (abgeleitet aus Lagemerkmalen und Entfernungen) basiert, nicht mehr ausreichend (7).

In marktwirtschaftlichen Systemen wird heute vor allem dem privatwirtschaftlichen Kalkuil der Nutzen- und Gewinnmaximierung eine hohe Bedeutung beigemessen, für das zwei Komponenten eine zentrale Rolle spielen:

die zu erzielende Bodenrente und

mögliche Agglomerationsvorteile.

Sie bestimmen die Nutzen-Kosten-Relation und nehmen somit Einfluss auf die Standortentscheidung von Unternehmen und Haushalten.

Voraussetzung für mögliche Investitionen ist zunächst die rechtliche und materielle Verfügbarkeit von Grund und Boden. In dieser Hinsicht bildet die verkehrliche Erschließung eine entscheidende Größe, da durch die Güte der Erreichbarkeit die Zentralität beeinflusst wird und somit auch die Bodenpreise. Damit wird im allgemeinen auch die zunehmende Dezentralisierung, also die Randwanderung von Haushalten und Unternehmen erklärt. Am Stadtrand oder im Umland sind zum einen Grundstïcke preiswerter und zum anderen können dort die gestiegenen Flächenansprüche eher verwirklicht werden. Die stattfindende Suburbanisierung verschärft sich noch durch die Konkurrenz von Kommunen um zusätzliche Steuereinnahmen.

Die Höhe der Bodenrente spiegelt die Knappheit des Bodens als Standort in Abhängigkeit vom urbanen Zentrum wider. Aufgrund der unterschiedlich hohen Grundrentenerwartungen unterschiedlicher städtischer Nutzungen differenziert sich die Stadtstruktur in Teilräume mit jeweils dominierenden Nutzungen. Dies macht die engen Zusammenhänge zwischen dem Wert eines Grundstïcks aufgrund der Zentralität seiner Lage (wie City, Peripherie, Stadtteilzentrum usw.), der Raumnutzung (Nutzungskonkurrenz) und der Stadtentwicklung deutlich. Die immer stärkere Differenzierung der Nutzungen in der Stadt(region) wird durch die zunehmende Heterogenität von Berufen, Waren und Dienstleistungen verstärkt.

Agglomerationsvorteile oder -nachteile beeinflussen die Entscheidung, inwieweit ein Unternehmen seine Standorte konzentrieren oder diversifizieren soll, sowie die Entscheidung, ob es seine Kapazität ausbauen oder verringern soll. Häufig werden Agglomerationswirkungen als Standortfaktor ohne genauere Spezifizierung neben andere Standortfaktoren, wie zum Beispiel Verkehrsanbindung, Rohstoffvorkommen oder Lohnniveau, gestellt. Der Begriff Agglomerationseffekt meint aber lediglich, dass die Qualität eines Unternehmensstandorts an der räumlichen Nähe zu anderen Unternehmen gemessen wird. Von „localization economies“ wird bei einer horizontalen Vernetzung gesprochen, also wenn sich Unternehmen einer Branche in einem Gebiet niederlassen. Von ,urbanization economies" dagegen wird bei einer vertikalen Konzentration von Unternehmen unterschiedlicher Branchen gesprochen.

\section{Ökonomische (Stadt-)Raum- wirkung auf der Mikroebene ...}

Auf der Mikroebene, also dem eigentlichen Standort eines Unternehmens, wirken vor allem Kosten für Flächen und Gebäude und deren Verfügbarkeit sowie Erreichbarkeit, Umfeldbedingungen und Agglomerationseffekte eng zusammen. Erreichbarkeit bedeutet hier vor allem

möglichst wenig Probleme bei der verkehrlichen Abwicklung am Standort, also ausreichende Rangiermöglichkeiten und Parkflächen (vielleicht auch das Vorhandensein eines Gleisanschlusses), und

möglichst geringe Nutzungskonflikte mit den Anliegern (Bewohner, Unternehmen), beispielsweise aufgrund von Lärmbelastungen und Schadstoffen infolge von Produktion und/oder Lieferund Kundenverkehren (Urbanisationsnachteile); aber auch

mögliche Fühlungsvorteile zu anderen wirtschaftlichen Akteuren (Unternehmen, Kunden). Sind Probleme auf der lokialen Ebene nicht lösbar, kommt es zur Abwanderung von Unterneh- men und Haushalten und zur Entmischung städtischer Nutzungen. Die Verdrängung anderer Nutzungen kann aber auch auf die Konzentrationstendenzen einzelner Nutzungen zurückgehen. Beispielsweise sind Unternehmen der Finanzdienstleistungen zum einen stark auf ,face-toface"-Kontakte angewiesen. Zum anderen sind sie in der Lage, einen hohen Umsatz pro Fläche zu erwirtschaften. Es verwundert daher nicht, dass sie es sind, die die zentralen Lagen in den Städten belegen.

\section{Mesoebene ...}

Auf der Mesoebene wirken aus stadt-ökonomischer Perspektive vor allem die Standortbedingungen im Wirkungs- und Verflechtungsraum der Stadtregion. Solange Standorte im engeren Verflechtungsraum oder im Umland der Städte als günstiger (Bodenpreise) oder besser erreichbar (Verkehrssystem) wahrgenommen werden, wird die Suburbanisierung anhalten und werden sich die Verkehrsprobleme verschärfen. Durch die Ökonomie kommen die Städte und das innerstädtische Verkehrssystem in direkte Konkurrenz mit ihrem Umland und dessen verkehrlicher Erreichbarkeit.

Der exzessive Ausbau der Verkehrs- und Kommunikationsinfrastruktur hat dazu beigetragen, dass Städte auch mit weit entfernt liegenden Städten immer mehr interagieren. Hier wirken Infrastrukturpolitik, Stadtentwicklung und Verkehrssystem eng zusammen: Schnellere Verkehrsmittel und/oder schnellere Straßen- oder Bahnverbindungen ermöglichen höhere Reisegeschwindigkeiten, so dass in gleicher Zeit weiter entfernt liegende Ziele erreicht werden können. Der Verkehrsaufwand steigt, die (empfundene) Reisezeit nicht. Ist die relative Kostensteigerung, z.B. als Anteil an den gesamten Haushaltsausgaben oder an den Gesamtkosten eines Produktes, ebenfalls nur gering, ist ein Verkehrswachstum vorprogrammiert.

\section{... und Makroebene}

Gleichzeitig fuihrt die Ausdifferenzierung der Wirtschaft sowie die weltweite Arbeitsteilung, die Zunahme transnationaler Konzerne und der Bedeutungsgewinn immaterieller Austauschbeziehungen zu Standortkonkurrenzen von (wenigen) Städten auf der Makroebene. Dies trifft vor allem auf diejenigen Städte zu, die in der Lage sind, wissensbasierte Wirtschaftsbereiche $\mathrm{zu}$ bündeln. Auf der Makroebene definiert sich Erreichbarkeit vor allem 
über die verkehrliche Anbindung einer Stadt an das Hochgeschwindigkeitsnetz der Bahnen und die Versorgung mit Flugrelationen und meint vor allem eine hohe Verkehrszentralität im Personenverkehr.

Die heute mögliche „grenzenlose“ Standortwahl von Unternehmen und Haushalten auf räumlicher Ebene führt zu starken räumlichen Disparitäten und regionalen Differenzierungen. Die sich ausbildenden spezifischen lokalen Wirtschafts- und Lebensverhältnisse liefern zugleich die Voraussetzungen für das Entstehen dieser selektiven Entwicklung. Oder wie Krätke formulierte: ,Städte müssen angesichts ihrer zunehmend grenzüberschreitenden Verflechtungsbeziehungen heute mehr denn je als ein System konkurrierender Standortzentren und Teil der Städtehierarchie betrachtet werden." (8) Die Internationalisierung von spezifischen Interaktionen und die räumliche Verteilung von Produktion und Dienstleistung wirkt damit auch direkt auf die lokale Ebene zurück.

\section{$>$ Fazit}

Die Städte befinden sich in einem mehrfachen Dilemma. Durch die heutigen gesellschaftlichen und wirtschaftlichen Veränderungsprozesse drohen sich die bisherigen urbanitätsbildenden Funktionen von Verkehrs- und Wirtschaftssystem gegen die Stadt zu richten. Weder gelingt in den Städten eine ausgewogene Funktionsmischung und verträgliche Dichte bei zugleich hoher Wohnqualität, noch eine polyzentrische Siedlungsstruktur in den Verdichtungsräumen oder eine dezentrale Konzentration der Siedlungen im ländlichen Raum. Vielmehr finden Konzentration und Dispersion gleichzeitig statt, mit hohen Anforderungen an das Verkehrssystem (Erreichbarkeit, Anschluss an die Verkehrsnetze). Dies geht häufig zu Lasten der (auch weiterhin gewünschten) Multifunktionalität von Städten und ist mit hohen negativen Urbanitätseffekten (Lärm, Stau, Luftbelastungen, erhöhte Bodenpreise) verbunden. Diesen funktionalen Zusammenhängen und räumlichen Wechselwirkungen von wirtschaftlichen und gesellschaftlichen sowie städtischen und verkehrlichen Prozessen wird heute jedoch in Theorie und Planungspraxis sowie in Wirtschaftsförderung und Ansiedlungspolitik zu wenig Bedeutung beigemessen.

Für die Stadtplanung und -politik besteht die Aufgabe, für die unterschiedliche Bedeutung von Städten im Städtenetz geeignete spezifische Strategien für die Stadt- und Verkehrsentwicklung zu entwerfen. Dafür müssen zwei Voraussetzungen gegeben sein. Erstens müssen Planung und Politik eine gewisse Offenheit für Innovationen an den Tag legen. Zweitens müssen die Anforderungen auf den unterschiedlichen räumlichen Ebenen in den Strategien Berücksichtigung finden. Im Idealfall sichern Stadtplanung und -politik die Multifunktionalität und die optimale Erreichbarkeit auch von innerstädtischen Standorten, ohne diese durch zusätzlichen Verkehr zu belasten (win-win-Situation).

Die einzelnen Systeme sind in ein dynamisches Umfeld eingebettet und werden von diesen Dynamiken wesentlich beeinflusst. Für die Stadtund Verkehrsforschung formuliert sich daraus der Anspruch, die zugrundeliegenden Systemdynamiken in ihren Wirkungen (Art, Richtungen und Intensität) grundlegend zu analysieren. Bisher fehlt es jedoch an geeigneten wissenschaftlichen und methodischen Grundlagen.

\section{Anmerkungen}

(1) Krätke, Stefan: Stadt - Raum - Ökonomie. Einführung in aktuelle Problemfelder der Stadtökonomie und Wirtschaftsgeographie. Basel/Boston/Berlin 1995, S. 9.

(2) Vgl. das laufende Forschungsvorhaben "Stadtentwicklungspotenziale und Verkehrswegeprojekte" in Auftrag des Bundesministeriums für Verkehr, Bauen und Wohnen, das vom IÖW gemeinsam mit der TransportTechnologie-Consult Karlsruhe und dem Institut für Regionalentwicklung und Strukturplanung in Erkner bearbeitet wird.

(3) Holz-Rou, Christian: Siedlungsstrukturen und Verkehr. Materialien zur Raumentwicklung, Bd. 84. Bonn 1997.

(4) Cooley, Charles H: The Theory of Transportation. Baltimore 1894.

(5) Wirth, Louis: Urbanism as a Way of Life. 1938.

(6) Friedrichs, Jürgen: Stadtsoziologie. Opladen 1995, S. $29 \mathrm{ff}$.

(7) Flämig, Heike/Markus Hesse: Der Verkehr als Gegenstand raumbezogener Forschung. Theoretische Überlegungen zur Bedeutung der Transport- und Transaktionskosten. Geographische Zeitschrift, 86. Jg. 1998, Heft 4, S. 225235.

(8) Krätke, Stefan: Stadtsystem im internationalen Kontext und Vergleich. In: Roth, Roland/Helmut Wollmann (Hrsg.): Kommunalpolitik, Politisches Handeln in den Gemeinden. Bonn 1993, S.176.

\section{Die Autorin}

Heike Flämig ist Leiterin des Forschungsfeldes Stadlentwicklung, Planung, Verkehr des Instituts für ökologische Wirtschaftsforschung

Kontakt: I0̈W, Potsdamer Str. 105, 10785 Berlin. Tel. 030/884594-19, Fax 030/ 8825439,

E-mail: Heike.Fluemig@ioew.de

\section{DER WEG IN DIE ÖKOLOGISCHE MODERNE}

\section{Hermann Scheer}

\section{SOLARE WELTWIRTSCHAFT}

Strategie für die ökologische Moderne

300 Seiten, DM 39,80

KUNSTMANN www.kunstmann.de

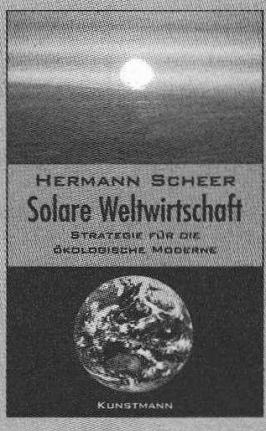

Nur eine Weltwirtschaft, die auf erneuerbaren Energien fußt, kann langfristig die Selbstzerstörung aller Wirtschafts- und Lebensformen verhindern.

In seinem neuen Buch zeigt Hermann Scheer die Strategie für den Weg in eine ökologische Moderne auf und entwickelt eine politische Ökonomie für ein solares Zeitalter. 
(c) 20I0 Authors; licensee IÖW and oekom verlag. This is an article distributed under the terms of the Creative Commons Attribution Non-Commercial No Derivates License (http://creativecommons.org/licenses/by-nc-nd/3.o/), which permits unrestricted use, distribution, and reproduction in any medium, provided the original work is properly cited. 